\title{
Successive interference cancelation and MAP decoding for mobile MIMO OFDM systems and their convergence behavior
}

\author{
Vamadevan Namboodiri $3^{*}$, Hong Liu ${ }^{2}$ and Predrag Spasojević ${ }^{1}$
}

\begin{abstract}
Turbo equalization schemes based on minimum mean square error criteria available in the literature for multiple-input multiple-output (MIMO) systems are computationally expensive, as they require a relatively large matrix inversion. In this article, we propose a suboptimal, successive interference cancelation (SIC)-based maximum a posteriori (MAP) decoding in doubly dispersive channels for orthogonal frequency division multiplexing (OFDM) MIMO systems (SIC-MAP-MIMO). SIC-MAP-MIMO leverages on the soft feedback symbol estimate to remove the intercarrier interference and coantenna interference from the received data thus making the subsequent MAP decoding simple. Extrinsic information transfer chart analysis supplemented with numerical simulation results show that SIC-MAP-MIMO achieves comparable BER performance to similar equalization schemes but with significant computational savings.
\end{abstract}

\section{Introduction}

Wireless communication based on MIMO systems has gained popularity due to the potential capacity increases it can provide [1]. OFDM has been a popular technique for transmission of signals over wireless channels primarily because the receiver design is relatively simple, as it does not require a complex equalizer. MIMOOFDM-based transmission systems can thus provide very high data rates with a relatively simple receiver design and are adopted in many recent wireless communication standards. Examples include (a) IEEE-802.11-n/ac [2,3], (b) IEEE $802.16 \mathrm{e} / \mathrm{m}$ (WiMAX) [4], and (c) LTE [5]. IEEE-802.11-n [2] specifies a maximum of $600 \mathrm{Mb} / \mathrm{s}$ using four independent spatial streams transmitted over a $40-\mathrm{MHz}$ channel. IEEE-802.11-ac [3] specifies a data rate of up to $3.5 \mathrm{~Gb} / \mathrm{s}$ using eight independent spatial streams in an $80-\mathrm{MHz}$ channel. WiMAX [4] specifies a limit of approximately $100 \mathrm{Mb} / \mathrm{s}$ using four spatial streams in a $5-\mathrm{MHz}$ channel. LTE suggests a peak data rate of $326.4 \mathrm{Mb} / \mathrm{s}$ using a $20-\mathrm{MHz}$ downlink with four transmit antennas [5]. Under static multipath channel conditions, the received signal in the MIMO receiver is corrupted only by coantenna interference (CAI). However,

\footnotetext{
*Correspondence: vnambood@winlab.rutgers.edu

3 Broadcom Corporation, Bangalore, India

Full list of author information is available at the end of the article
}

high transceiver mobility at high carrier frequency causes severe time-varying frequency-selective multipath fading at the receiver. This breaks the orthogonality of subcarriers and hence causes intercarrier interference (ICI) in the received signal. As an example, at a transmission frequency of $5 \mathrm{GHz}$ and at vehicular speeds of 240 $480 \mathrm{~km} / \mathrm{h}$, which are common in high-speed trains, the expected maximum receiver Doppler spread in WiMAX and LTE systems is of the order of 12 to $23 \%$ of the intercarrier spacing. Furthermore, it is believed that future wireless communications will adopt higher carrier frequencies and higher mobility requirements, further increasing the maximum relative Doppler frequency and exacerbating the ICI. In such scenarios, as discussed in this article, the efficient detector design for MIMOOFDM systems is a challenging practical problem.

Some early equalization schemes proposed in the literature to cope with ICI and CAI are (a) block linear [6], (b) banded minimum mean square error (MMSE) linear [7], and (c) banded MMSE decision-feedback [8]. More recently, various iterative equalization schemes based on successive cancelation of ICI and CAI [9-13] or turbo principle $[9,14-16]$ were proposed. A brief survey of the iterative equalization schemes published in the last decade and how they differ from the proposed scheme is given in the sequel. In general, turbo-like iterative schemes are 
found to have superior performance compared to others, but they usually suffer from high computation complexity, albeit at varying degrees, and thus require high silicon area for implementation and high battery power for operation. Such practical application challenges have motivated us to propose a new low-complexity detector scheme for OFDM-MIMO with an improved trade-off between performance and implementation complexity in [17] and in this study.

The iterative/successive interference cancelation scheme proposed in Section "SIC-based MAP receiver: MIMO (SIC-MAP-MIMO)" is related to, yet distinct from, a number of published algorithms. In [9], multiple access interference (MAI) and inter-symbol interference (ISI) in a static multipath environment are removed in a code-division-multipath-access (CDMA) system using a combination of soft-interference cancelation and linear MMSE filtering. [10] is an extension of the scheme proposed in [9], but in [10], additional filtering is performed to suppress both the ISI and MAI residuals. Turbo Equalization (TE) proposed in [18] performs MMSE-based turbo estimation of the transmitted symbol on singlecarrier systems under static channel conditions, followed by LLR computation and BCJR decoding. This involves matrix inversion for the estimation of every symbol per iteration and is thus computationally expensive $\left(O\left(N^{2}\right)\right.$ operations). Additional complexity reduction for TE is achieved in doubly selective OFDM systems by working on a submatrix around the system matrix as in [19]. SICMAP-MIMO is perhaps close to the ISI cancelation stage of [9]. However, unlike [9,10], SIC-MAP-MIMO requires only $O(N)$ operations. It leverages the banded sparse structure of the single-user LTV MIMO system matrix, where the significant channel coefficients are concentrated in a banded structure along the diagonal $[6,8,19]$ as shown in Figure 1 (right). There are a number of SIC schemes which try to diagonalize the system matrix. In [11], an iterative decision feedback equalizer is proposed to perform ICI cancelation such that the modified system matrix becomes diagonal and, consequently, the equalizer becomes single-tap. In [20], ICI is removed from the time domain signal (resulting in a diagonal frequency domain system matrix) and is converted to frequency domain. Hard decisions are made on the equalized signal, following which it is converted back to time domain and the time-frequency iterations are repeated. In [12], the mean value of the transmit symbol is computed using the LLR values from the decoder. This is used to remove the ICI from the received symbol, resulting in a diagonal system matrix. A modified low-complexity MMSE equalizer that takes the decision error into account is now derived. In [13], a turbo-EM receiver is proposed. Here, the system matrix is estimated from the EM detector, whereas transmit symbols are estimated either using the
EM algorithm or from the LLR values from the decoder. Using these estimates, ICI is computed and removed as in $[11,12]$ to obtain a diagonal system matrix. The scheme in [21] is applicable to single-carrier (SC) systems. Here, the received signal is split into small segments, such that the channel remains approximately static during each small segment. Suitable signal processing is performed on each of these segments such that the resulting channel matrix is made diagonal. TE, like the one described in [18], is performed on the modified system to recover the received bits. The above-described schemes try to obtain a modified system with only diagonal entries, such that a single tap equalizer can equalize the modified system. Unlike this, in SIC-MAP-MIMO, copies of the received signal on the same and adjacent subcarriers of all receive antennas are carefully separated out to obtain frequency diversity. The resulting system matrix is a column matrix. It has been identified through simulations that the banded sparse structure of the system matrix, as in the case of doubly selective MIMO channels, allows this simplification without sacrificing performance. MAP decoding is performed on this simplified system. The scheme proposed in [15] is similar to [19], but is extended to OFDM MIMO. It proposes a new window for received signal. SIC-MAP-MIMO does not perform windowing, but better performance can be expected with any of the windowing proposed above.

In this article, we propose a suboptimal, SIC-based MAP decoder. In an OFDM-MIMO system operating in a doubly selective environment, a QAM symbol transmitted from a particular subcarrier of a given antenna spreads to the same and adjacent subcarriers of all receive antennas, causing ICI and CAI. In SIC-MAP-MIMO, copies of the received signal on the same and adjacent subcarriers of all receive antennas are carefully separated out, as in the case of a frequency diversity system. CAI and ICI, if they exist, are estimated iteratively using the conditional symbol mean estimates obtained from the decoder feedback information from the previous iteration. These estimates are removed appropriately from the received symbol. Hence the resulting system matrix becomes a single-column matrix. MAP decoding of the resulting system is simple to implement. Motivated from $[15,19,22]$, we exploit the banded nature of the system matrix in SIC-MAP-MIMO. The performance and computational complexity of the proposed scheme are compared with schemes suggested in $[19,22]$ when extended to MIMO. It has been found that SIC-MAP-MIMO provides a comparable performance to the above schemes, but with significantly less computational complexity, making it especially suitable for mobile applications where battery power is limited. Convergence behavior of the above schemes is also analyzed using extrinsic information transfer (EXIT) charts [23]. 


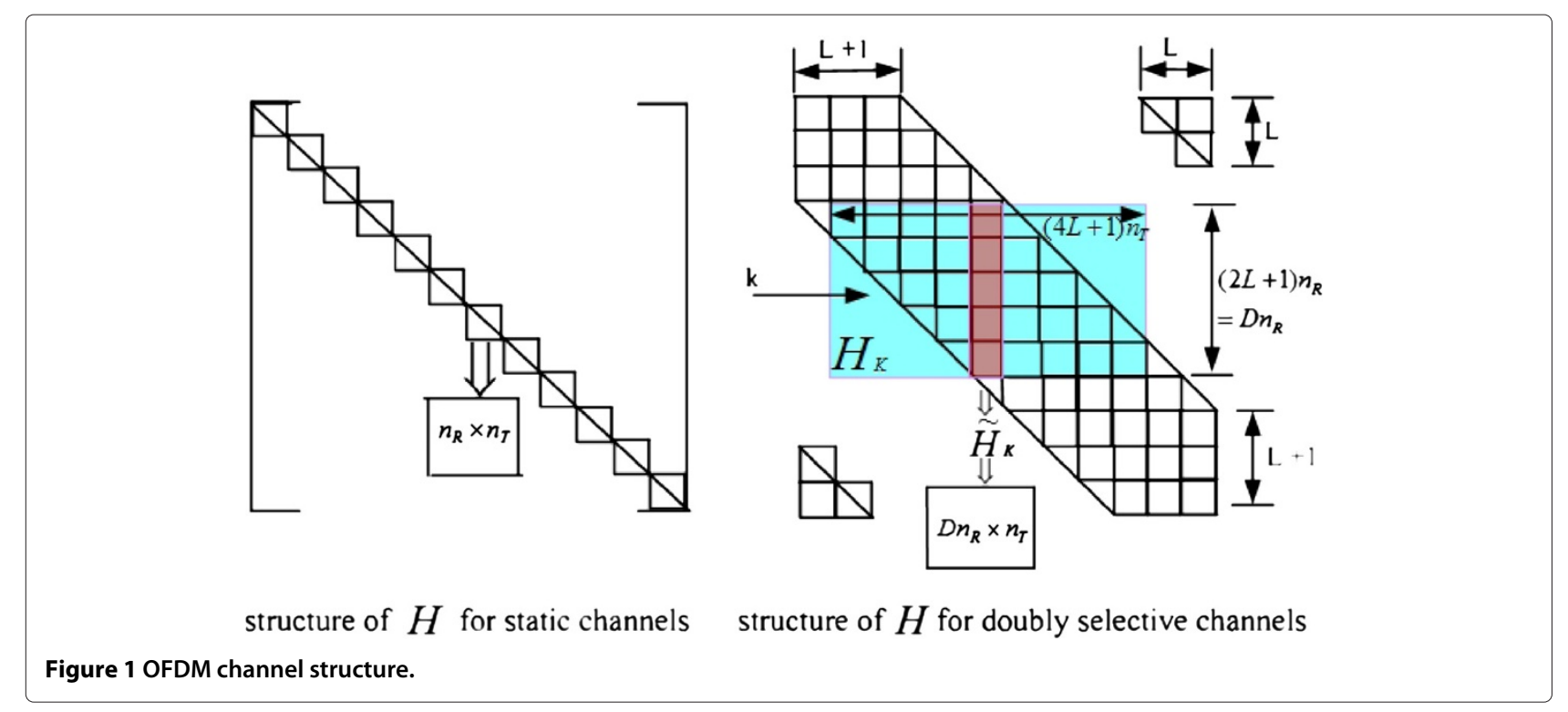

This article is organized as follows: Notations used in this article are explained first. In the next section, the system model is presented, followed by a description of SIC-MAP-MIMO in Section "SIC-based MAP receiver: MIMO (SIC-MAP-MIMO)". In Section "Computational complexity analysis", we compare the computation complexity of SIC-MAP-MIMO with similar equalization schemes. In Section "Numerical results", the numerical results are presented. The article concludes with "Conclusion" section, where we draw final conclusions.

Notation: $(\cdot)^{t}$ denotes transpose; $(\cdot)^{H}$ denotes conjugate transpose (Hermitian); $\otimes$ is the Kronecker product; $\{a\}$ denotes a set with elements $\{a(0), a(1), \ldots\}$; F for normalized $N$ point Discrete Fourier Transform (DFT), where $\mathbf{F}_{k, l}:=(1 / \sqrt{N}) e^{-j 2 \pi k l / N} ; \mathbf{I}$ is the identity matrix; $\mathbf{i}_{k}$ is the $k^{\text {th }}$ column of $\mathbf{I} ; \mathbf{0}_{n_{R} \times n_{T}}$ is the null matrix of size $n_{R} \times n_{T} ; *$ denotes convolution; $\|\cdot\|$ for $l_{2}$-norm; $\lceil\cdot\rceil$ is the ceiling of a function; modulo- $N$ is denoted by $\langle\cdot\rangle_{N}$; $\operatorname{Re}(\cdot)$ and $\operatorname{Im}(\cdot)$ for the real and imaginary parts, respectively. $\operatorname{diag}\left(\boldsymbol{v}_{\mathbf{x}}\right)$ is the diagonal matrix with vector $\boldsymbol{v}_{\mathbf{x}}$ in the main diagonal. Expectation is denoted by $E\{\cdot\}$. Both $x$ and $\cdot$ are used to denote multiplication. Bold lowercase letters (e.g., $\mathbf{x}$ ) denote vectors, and bold uppercase letters (e.g., $\mathbf{X})$ denote matrices. Covariance is denoted by $\operatorname{cov}(\mathbf{b}, \mathbf{c}):=E\left\{\mathbf{b} \mathbf{c}^{H}\right\}-E\{\mathbf{b}\} E\left\{\mathbf{c}^{H}\right\}$.

\section{System model}

The MIMO OFDM transceiver system with $n_{T}$ transmit and $n_{R}$ receive antennas used in this article is given in Figure 2 . We assume that $n_{T} \leq n_{R}$. Information bits $(\{a\})$ are convolutionally encoded $(\{b\})$ and passed through a bit interleaver $(\{c\})$. The symbol mapper modulates them into QAM symbols $(\{s\})$. A set of $N$ of these coded QAM "frequency domain" symbols is collected to form an OFDM symbol. The demultiplexer collects $n_{T}$ OFDM symbols (an OFDM symbol frame) and sends each symbol ( $\left.\left\{\mathbf{s}_{\mathbf{q}}\right\}\right)$ to one of the $n_{T}$ transmit paths. The symbol interleaver (SI) in each path interleaves them $\left(\left\{\mathbf{x}_{q}\right\}\right)$. They are then converted into "discrete time-domain" samples $\left(\left\{\mathbf{z}_{q}\right\}\right)$ by performing an $N$ - point IDFT. A cyclic prefix (CP) of length $N_{p} \leq N$ is added to each of these symbols. They are then simultaneously transmitted from $n_{T}$ transmit antennas. Transmit and receive antennas are assumed to be placed sufficiently far apart among themselves so that the $n_{T} \cdot n_{R}$ multipath channels are independent. Furthermore, these channels are assumed to be both frequency- and time-selective and are modeled as a linear time-varying (LTV) system with a discrete impulse response $h_{p q}(i, l)$ that is defined as the time $i$ response to an impulse at time $i-l$ for the wireless channel from the $q$ th transmit antenna to the $p$ th receive antenna. Static multipath channel conditions are treated as a special case of the above general formulation. At the receiver, the CP-removed OFDM data from each receive antenna are converted back to the "frequency domain" by performing $N$-point DFT and passed to the SIC and Symbol Deinterleaver. The log likelihood ratio (LLR) computer computes the LLRs of the received bits from the interference removed observation. This is appropriately multiplexed, bit-deinterleaved, and passed to a BCJR- or SOVA-based decoder.

We assume perfect carrier, symbol, and sample synchronization at the receiver. Besides, it is assumed that the channel is known at the receiver. We follow the modeling used in [6]. Assuming that maximum channel delay spread $N_{h} \leq N_{p}$, the received samples on any of the $p$ receive antennas in the baseband can be represented as 


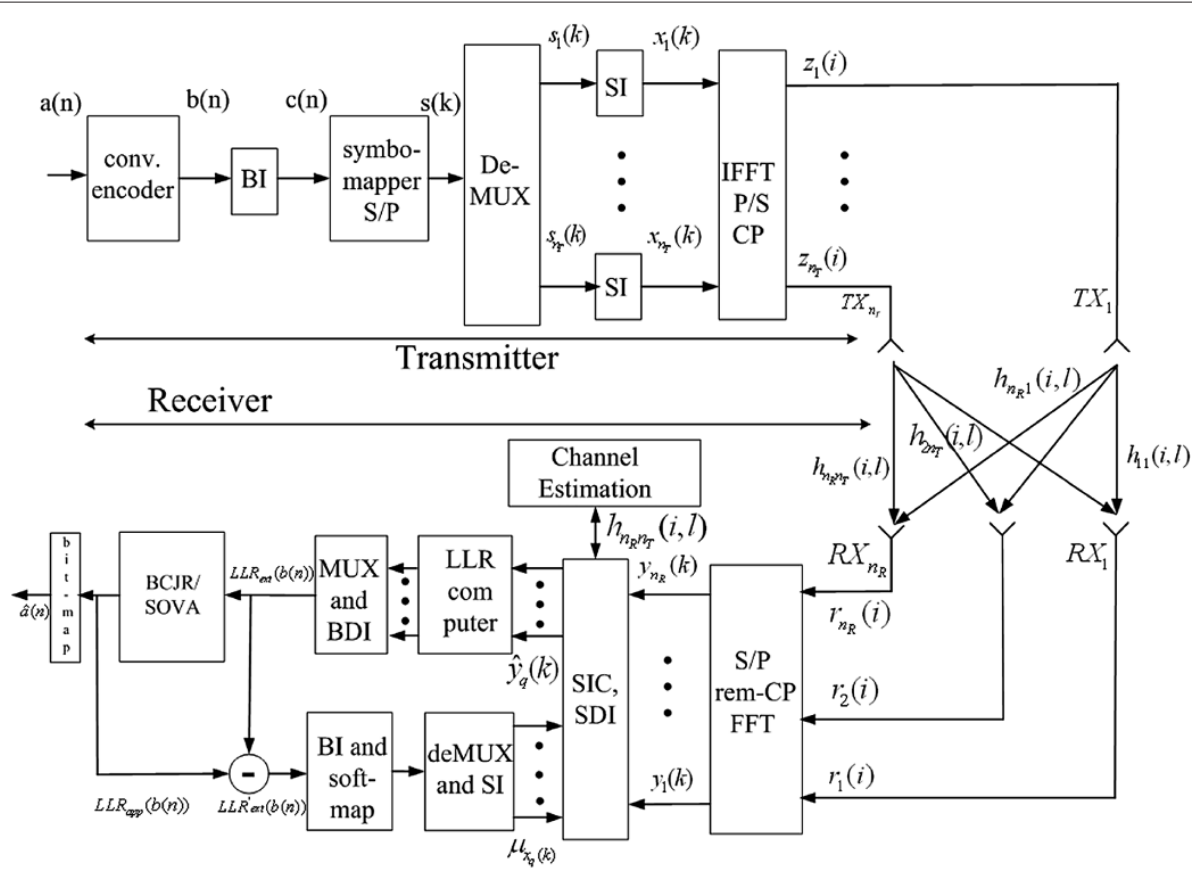

Figure 2 MIMO OFDM transceiver.

$$
r_{p}(i)=\sum_{q=1}^{q=n_{T}} \sum_{l=0}^{l=N_{h}-1} h_{p q}(i, l) z_{q}(i-l)+n_{p}(i), 0 \leq i<N,
$$

where $\left\{n_{p}(i)\right\}$ are additive white Gaussian noise (AWGN) samples on the $p$ th receive antenna with zero mean and variance $\sigma^{2}$ (we assume equal noise power on all receive antennas). The condition $N_{h} \leq N_{p}$ ensures that $r_{p}(i)$ contains contributions only from the currently transmitted OFDM symbol frame. The received vector at the $i$ th time instant, $\mathbf{r}(i):=\left[r_{1}(i), r_{2}(i), \ldots, r_{n_{R}}(i)\right]^{t}$, can be expressed as

$$
\mathbf{r}(i)=\sum_{l=0}^{l=N_{h}-1} \mathcal{H}(i, l) \mathbf{z}(i-l)+\mathbf{n}(i), 0 \leq i<N,
$$

where

$$
\mathcal{H}(i, l):=\left[\begin{array}{llll}
h_{11}(i, l) & h_{12}(i, l) & \ldots & h_{1 n_{T}}(i, l) \\
h_{21}(i, l) & h_{22}(i, l) & \ldots & h_{2 n_{T}}(i, l) \\
\ldots & \ldots & \ldots & \ldots \\
h_{n_{R} 1}(i, l) & h_{n_{R} 2}(i, l) & \ldots & h_{n_{R} n_{T}}(i, l)
\end{array}\right],
$$

$\mathbf{z}(i):=\left[z_{1}(i), z_{2}(i), \ldots, z_{n_{T}}(i)\right]^{t}$ and $\mathbf{n}(i):=\left[n_{1}(i), n_{2}(i)\right.$, $\left.\ldots, n_{n_{R}}(i)\right]^{t}$. Over a time window of $\mathrm{N}$ sample duration, (2) can be expressed in matrix form as

$$
\mathbf{r}=\mathbf{\Xi} \mathbf{z}+\psi
$$

where $\mathbf{r}:=\left[\mathbf{r}^{t}(0), \mathbf{r}^{t}(1), \ldots, \mathbf{r}^{t}(N-1)\right]^{t} \in \mathcal{C}^{N \cdot n_{R}}, \mathbf{z}:=\left[\mathbf{z}^{t}(0)\right.$, $\left.\mathbf{z}^{t}(1), \ldots, \mathbf{z}^{t}(N-1)\right]^{t} \in \mathcal{C}^{N \cdot n_{T}}, \boldsymbol{\psi}:=\left[\mathbf{n}^{t}(0), \mathbf{n}^{t}(1), \ldots, \mathbf{n}^{t}\right.$
$(N-1)]^{t} \in \mathcal{C}^{N \cdot n_{R}}$ and $\boldsymbol{\Xi} \in \mathcal{C}^{N \cdot n_{R} \times N \cdot n_{T}}$ is the time varying system matrix given in (4).

$$
\Xi:=\left[\begin{array}{cccccc}
\mathcal{H}(0,0) & \mathbf{0}_{n_{R} \times n_{T}} & \ldots & \mathcal{H}\left(0, N_{h}-1\right) & \ldots & \mathcal{H}(0,1) \\
\mathcal{H}(1,1) & \mathcal{H}(1,0) & \mathbf{0}_{n_{R} \times n_{T}} & \ldots & \ldots & \mathcal{H}(1,2) \\
\ldots & \ldots & \ldots & \ldots & \ldots & \ldots \\
\mathbf{0}_{n_{R} \times n_{T}} & \ldots & \mathcal{H}\left(N-1, N_{h}-1\right) & \ldots & \ldots & \mathcal{H}(N-1,0)
\end{array}\right]
$$

$N$ samples from the same OFDM symbol from $n_{R}$ receive antennas (a total of $N \cdot n_{R}$ samples) are grouped together and presented to a DFT processor which, in turn, outputs $N \cdot n_{R}$ "frequency domain" samples. This operation can be represented mathematically as follows:

$$
\begin{aligned}
\mathbf{y} & =\mathbf{Q}^{(R x)} \mathbf{r}=\mathbf{Q}^{(R x)} \mathbf{\Xi} \mathbf{z}+\mathbf{Q}^{(R x)} \boldsymbol{\psi} \\
& =\mathbf{Q}^{(R x)} \mathbf{\Xi} \mathbf{Q}^{(T x) H} \mathbf{x}+\mathbf{w} \\
& =\mathbf{H} \mathbf{x}+\mathbf{w},
\end{aligned}
$$

where $\mathbf{Q}^{(T x)}=\mathbf{F} \otimes \mathbf{I}_{n_{T}}, \mathbf{Q}^{(R x)}=\mathbf{F} \otimes \mathbf{I}_{n_{R}}, \mathbf{H}=\mathbf{Q}^{(R x)} \mathbf{\Xi} \mathbf{Q}^{(T x) H}$, $\mathbf{z}=\mathbf{Q}^{(T x) H} \mathbf{x}, \mathbf{y}:=\left[\mathbf{y}^{t}(0), \mathbf{y}^{t}(1), \ldots, \mathbf{y}^{t}(N-1)\right]^{t}, \mathbf{y}(k):=$ $\left[y_{1}(k), y_{2}(k), \ldots, y_{n_{R}}(k)\right]^{t}, \mathbf{x}:=\left[\mathbf{x}^{t}(0), \mathbf{x}^{t}(1), \ldots, \mathbf{x}^{t}(N-\right.$ $1)]^{t}, \mathbf{x}(k):=\left[x_{1}(k), x_{2}(k), \ldots, x_{n_{T}}(k)\right]^{t}$ and $\mathbf{w}=\mathbf{Q}^{(R x)} \psi$. Note that (a) each element of $\mathbf{H}$ can be written as,

$$
\begin{aligned}
H_{p q}(m, n)= & \frac{1}{N} \sum_{l=0}^{l=N_{h}-1} \sum_{i=0}^{i=N-1} \\
& \times h_{p q}(i, l) e^{-j 2 \pi(m-n) i / N} e^{-j 2 \pi l n / N}
\end{aligned}
$$

and (b) $\mathbf{w}$ is wide sense stationary (WSS) with the mean and the covariance identical to that of $\psi$, since $F$ is unitary. 
The total transmit power is assumed to be unity with all antennas transmitting equal power. Multipath timevarying channel coefficients are modeled as zero mean complex Gaussian random variables. Fading coefficients for different paths are assumed to be statistically independent while the coefficient for a given path is timecorrelated with the autocorrelation function (wide-sense stationary uncorrelated scattering model) given by [24],

$$
E\left\{h(m, l) h(n, l)^{*}\right\}=\alpha_{l} \mathcal{J}_{0}\left(2 \pi(m-n) f_{d} T_{s}\right)
$$

where $\alpha_{l}$ is the average power of the $l$ th path, $\mathcal{J}_{0}(\cdot)$ is the zeroth-order Bessel function of the first kind, $T_{S}$ is the sampling interval, and $f_{d}$ is the maximum Doppler frequency given by

$$
f_{d}=\frac{v}{c} f_{c} \cos \left(\theta_{d}\right)
$$

Here $v$ is the vehicle speed, $c$ is the velocity of light, $f_{c}$ is the carrier frequency, and $\theta_{d}$ is the scattering angle.

It has been shown that $\mathbf{H}$ will be a block-banded matrix with significant block coefficients concentrated in a banded structure, with width $D$ along the diagonal $[6,8,19]$. $D$ is a design parameter typically chosen as $D=$ $2 L+1$, where $L=\left\lceil f_{d} T_{s} N\right\rceil$ in which $N$ is the OFDM symbol length. If the channel is static, $\Xi$ will be a block circulant matrix and $\mathbf{H}$ will be a block diagonal matrix. Different structures of $\mathbf{H}$ are shown in Figure 1. Interference from adjacent subcarriers gives raise to ICI. The received signal on each subcarrier at each receiver antenna contains contributions from all transmit antennas. This gives rise to CAI [6].

\section{SIC-based MAP receiver: MIMO (SIC-MAP-MIMO)}

\section{Formulation of the proposed MAP receiver}

In this section, we present a low-complexity iterative receiver that implements $\mathrm{SIC}$, followed by MAP decoding for MIMO systems. The proposed scheme first modifies the system matrix to a single column matrix by selectively removing the ICI and CAI interference from the received symbols, where ICI and CAI interference are computed using the feedback symbol mean values. Soft information can be computed directly with low cost from this modified model. These are fed to a MAP bit decoder. The following observations are key in formulating the proposed scheme:

1. The relative magnitude of each subblock and superblock diagonal element of the doubly selective Rayleigh fading channel matrix $\mathbf{H}$ decreases significantly as we move away from the main diagonal. This has been justified in $[19,22]$. We can thus ignore all elements that are far away from the main diagonal without significantly impacting performance. This is further justified through simulations in the "Numerical results" section. Note that these elements are absent for a static multipath channel.

2. As the extrinsic information becomes more accurate over multiple turbo iterations, the conditional mean, $\boldsymbol{\mu}_{\mathbf{x}}(k) \rightarrow \mathbf{x}(k)$, which is the true symbol value and the conditional variance, $\boldsymbol{v}_{\mathbf{x}}(k) \rightarrow \mathbf{0}_{n_{T} \times 1}$.

Therefore, in each new iteration we can use $\boldsymbol{\mu}_{\mathbf{x}}(k)$ from the previous iteration to selectively remove CAI and ICI from the received symbol in such a manner that the resulting system matrix is turned into a column matrix. MAP decoding of the modified system is computationally efficient to implement.

Based on observation 1, (5) can be approximated as

$$
\begin{aligned}
\mathbf{y}_{\mathbf{k}} & :=\left[\mathbf{y}\left(\langle k-L\rangle_{N}\right), \ldots, \mathbf{y}\left(\langle k+L\rangle_{N}\right)\right]^{t} \\
& =\mathbf{H}_{\mathbf{k}} \mathbf{x}_{\mathbf{k}}+\mathbf{w}_{\mathbf{k}},
\end{aligned}
$$

where $\mathbf{x}_{\mathbf{k}}:=\left[\mathbf{x}\left(\langle k-2 L\rangle_{N}\right), \ldots, \mathbf{x}\left(\langle k+2 L\rangle_{N}\right)\right]^{t}, \mathbf{w}_{\mathbf{k}}:=$ $\left[\mathbf{w}\left(\langle k-L\rangle_{N}\right), \ldots, \mathbf{w}\left(\langle k+L\rangle_{N}\right)\right]^{t}$, and $\mathbf{H}_{\mathbf{k}}$ is the shaded (green) section of $\mathbf{H}$ in Figure 1 (right) given by (10). Note that modulo- $N\left(\langle\rangle_{N}\right)$ operation is used in the above equation, thanks to the $\mathrm{CP}$ in the system.

$\mathbf{H}_{\mathbf{k}}:=\left[\begin{array}{ccc}\mathbf{H}\left(\langle k-L\rangle_{N},\langle k-2 L\rangle_{N}\right) & \ldots & \mathbf{H}\left(\langle k-L\rangle_{N},\langle k+2 L\rangle_{N}\right) \\ \mathbf{H}\left(\langle k-L+1\rangle_{N},\langle k-2 L\rangle_{N}\right) & \ldots & \mathbf{H}\left(\langle k-L+1\rangle_{N},\langle k+2 L\rangle_{N}\right) \\ \ldots & \ldots & \ldots \\ \mathbf{H}\left(\langle k+L\rangle_{N},\langle k-2 L\rangle_{N}\right) & \ldots & \mathbf{H}\left(\langle k+L\rangle_{N},\langle k+2 L\rangle_{N}\right)\end{array}\right]$

Each element $\mathbf{H}(m, n)$ in (10) (one small grid in Figure 1) is itself a matrix of size $n_{R} \times n_{T}$ given as

$$
\mathbf{H}(m, n):=\left[\begin{array}{ccc}
H_{11}(m, n) & \ldots & H_{1 n_{T}}(m, n) \\
\ldots & \ldots & \ldots \\
H_{n_{R} 1}(m, n) & \ldots & H_{n_{R} n_{T}}(m, n) .
\end{array}\right]
$$

For simplicity of notation, the modulo operation $\left(\langle\rangle_{N}\right)$ is omitted in the sequel. Now, $\mathbf{x}_{\mathbf{k}}=\boldsymbol{\mu}_{\mathbf{x}_{\mathbf{k}}}+\boldsymbol{\delta}_{\mathbf{x}_{\mathbf{k}}}$, where $\boldsymbol{\delta}_{\mathbf{x}_{\mathbf{k}}}$ is the residual error, which approaches $\mathbf{0}_{4 L+1}$ as the extrinsic LLR becomes more reliable over multiple iterations. Substituting for $\mathbf{x}_{\mathbf{k}}$ in (9) and rearranging yields (12), $\tilde{\mathbf{w}}_{\mathbf{k}}$, the new noise, contains the ICI from the residual error $\delta_{\mathbf{x}_{\mathbf{k}}}$. $\tilde{\mu}_{\mathbf{x}_{\mathbf{k}}}$ is as defined in (12). 


$$
\begin{aligned}
\mathbf{y}_{\mathbf{k}}=\mathbf{H}_{\mathbf{k}}\left[\mathbf{i}_{2 L \cdot n_{T}}, \ldots, \mathbf{i}_{(2 L+1) \cdot n_{T}-1}\right] \mathbf{x}(k)+\mathbf{H}_{\mathbf{k}}\left[\begin{array}{c}
\boldsymbol{\mu}_{\mathbf{x}}(k-2 L) \\
\cdot \\
\mathbf{0}_{n_{T}} \\
\cdot \\
\boldsymbol{\mu}_{\mathbf{x}}(k+2 L)
\end{array}\right] \\
+\underbrace{\mathbf{H}_{\mathbf{k}}\left[\begin{array}{c}
\delta_{\mathbf{x}}(k-2 L) \\
\cdot \\
\mathbf{0}_{n_{T}} \\
\cdot \\
\delta_{\mathbf{x}}(k+2 L)
\end{array}\right]+\mathbf{w}_{\mathbf{k}}}_{\tilde{\mu}_{x_{k}}}
\end{aligned}
$$

Let

$$
\begin{aligned}
\tilde{\mathbf{y}}_{\mathbf{k}} & :=\mathbf{y}_{\mathbf{k}}-\mathbf{H}_{\mathbf{k}} \tilde{\mu}_{\mathbf{x}_{\mathbf{k}}} \\
& =\mathbf{H}_{\mathbf{k}}\left[\mathbf{i}_{2 L \cdot n_{T}}, \ldots, \mathbf{i}_{(2 L+1) \cdot n_{T}-1}\right] \mathbf{x}(k)+\tilde{\mathbf{w}}_{\mathbf{k}} \\
& =\tilde{\mathbf{H}}_{\mathbf{k}} \mathbf{x}(k)+\tilde{\mathbf{w}}_{\mathbf{k}} .
\end{aligned}
$$

Notice that $\tilde{\mathbf{y}}_{\mathbf{k}} \in \mathcal{C}^{D \cdot n_{R}}$ and $\mathbf{x}(k) \in \mathcal{C}^{n_{T}}$ and $\tilde{\mathbf{H}}_{\mathbf{k}}$ are shown in red in Figure 1 (right). It is a matrix of size $D \cdot n_{R} \times n_{T}$. For static channels where $L=0$, $\tilde{\mathbf{y}}_{\mathbf{k}}$ will only have $n_{R}$ non-zero elements at the center (Figure 1, left). While dealing with the reception of $x_{q}(k)$, the $k$ th symbol from the $q$ th transmit antenna, $k$ th symbols from all other transmit antennas $\left(\left\{x_{l}(k)_{l \neq q}\right\}\right)$ are causing CAI on the received samples $\mathbf{y}_{\mathbf{k}}$. Using similar techniques to those given above, the CAI can be estimated and removed from the system as well. The resulting system equation is

$$
\mathbf{y}_{\mathbf{q}_{\mathbf{k}}}^{\prime}=\mathbf{h}_{\mathbf{q}_{\mathbf{k}} x_{q}}(k)+\mathbf{w}_{\mathbf{q}_{\mathbf{k}}}^{\prime},
$$

where $\mathbf{y}_{\mathbf{q}_{\mathbf{k}}}^{\prime}:=\tilde{\mathbf{y}}_{\mathbf{k}}-\tilde{\mathbf{H}}_{\mathbf{k}} \tilde{\mu}_{\mathbf{x}_{\mathbf{q}}}(k), \mathbf{h}_{\mathbf{q}_{\mathbf{k}}}:=\tilde{\mathbf{H}}_{\mathbf{k}} i_{q}, \tilde{\mu}_{\mathbf{x}_{\mathbf{q}}}(k):=\left[\mu_{x_{1}}\right.$ $\left.(k), \ldots, \mu_{x_{q-1}}(k), 0, \mu_{x_{q+1}}(k), \ldots, \mu_{x_{n_{T}}}(k)\right]^{t} \quad$ and $\quad \mathbf{w}_{\mathbf{q}_{\mathbf{k}}}^{\prime}:=$ $\tilde{\mathbf{w}}_{\mathbf{k}}+\tilde{\mathbf{H}}_{\mathbf{k}} \tilde{\delta}_{\mathbf{x}_{\mathbf{q}}}(k)$, where $\tilde{\delta}_{\mathbf{x}_{\mathbf{q}}}(k):=\left[\delta_{x_{1}}(k), \ldots, \delta_{x_{q-1}(k)}, 0\right.$, $\left.\delta_{x_{q+1}}(k), \ldots, \delta_{x_{n_{T}}}(k)\right]^{t}$. We assume $\mathbf{w}_{\mathbf{q}_{\mathbf{k}}}^{\prime}$ has a variance of $\sigma^{\prime 2} I_{(2 L+1) n_{R}}$. As noted earlier and as will be shown later in Section "Numerical results", the combined contributions of residual ICI and CAI to the noise variance $\sigma^{\prime 2}$ are small and decreasing over multiple iterations as the reliability in the feedback information increases. We thus approximate $\sigma^{\prime 2} \mathbf{I}_{n_{R}(2 L+1)} \approx \sigma^{2} \mathbf{I}_{n_{R}(2 L+1)}$.

The LLR computer calculates $L L R_{\text {ext }}\left(c_{q}(n)\right)$, the extrinsic LLR. It represents information about $c_{q}(n)$ contained in $\mathbf{y}_{\mathbf{q}_{\mathbf{k}}}^{\prime}$ and $P\left(c_{q}(l)\right)$ for all $l \neq n$. These are passed to a MAP decoder where they are used as $a$ priori LLRs. $\operatorname{LLR}_{\text {ext }}\left(c_{q}(n)\right)$ is calculated from the modified system using (15), where $0 \leq i \leq Q-1, \mathcal{S}=\left[m_{0}\right.$, $\left.m_{1}, \ldots, m_{Q-1}\right]^{t} \in F_{2},\{\eta\}=\operatorname{map}(\mathcal{S})$ is the signal constellation and $F_{2}$ is binary Galois Field. $Q$ denotes the number of bits per symbol. For example, $Q=1$ for BPSK, $Q=2$ for QPSK, and so on.

$$
\begin{aligned}
\operatorname{LLR}_{\text {ext }}\left(c_{q}(Q k+i)\right) \\
=\operatorname{LLR}_{\mathrm{app}}\left(c_{q}(Q k+i)\right)-\operatorname{LLR}\left(c_{q}(Q k+i)\right) \\
=\ln \frac{P\left(\left(c_{q}(Q k+i)=0\right) \mid \mathbf{y}_{\mathbf{q}_{\mathbf{k}}}^{\prime}\right)}{P\left(\left(c_{q}(Q k+i)=1\right) \mid \mathbf{y}_{\mathbf{q}_{\mathbf{k}}}^{\prime}\right)}-\operatorname{LLR}\left(c_{q}(Q k+i)\right) \\
=\ln \frac{p\left(\mathbf{y}_{\mathbf{q}_{\mathbf{k}}}^{\prime} \mid\left(c_{q}(Q k+i)=0\right)\right) P\left(c_{q}(Q k+i)=0\right)}{p\left(\mathbf{y}_{\mathbf{q}_{\mathbf{k}}}^{\prime} \mid\left(c_{q}(Q k+i)=1\right)\right) P\left(c_{q}(Q k+i)=1\right)} \\
\quad-\operatorname{LLR}\left(c_{q}(Q k+i)\right) \\
=\left(\ln \frac{p\left(\mathbf{y}_{\mathbf{q}_{\mathbf{k}}}^{\prime} \mid\left(c_{q}(Q k+i)=0\right)\right)}{p\left(\mathbf{y}_{\mathbf{q}_{\mathbf{k}}}^{\prime} \mid\left(c_{q}(Q k+i)=1\right)\right)}+\operatorname{LLR}\left(P\left(c_{q}(Q k+i)\right)\right)\right. \\
\quad-\operatorname{LLR}\left(c_{q}(Q k+i)\right) \\
=\ln \frac{\sum_{\mathcal{S} \in F_{2}: \mathcal{S}_{i}=0} p\left(\mathbf{y}_{\mathbf{q}_{\mathbf{k}}}^{\prime} \mid\left(x_{q}(k)=\operatorname{map}(\mathcal{S})\right)\right) \prod_{j=0: j \neq i}^{Q-1} P\left(m_{j}\right)}{\sum_{\mathcal{S} \in F_{2}: \mathcal{S}_{i}=1} p\left(\mathbf{y}_{\mathbf{q}_{\mathbf{k}}}^{\prime} \mid\left(x_{q}(k)=\operatorname{map}(\mathcal{S})\right)\right) \prod_{j=0 ; j \neq i}^{Q-1} P\left(m_{j}\right)}
\end{aligned}
$$

As shown in the Appendix, for QPSK, the above expression can be simplified as

$$
\begin{aligned}
\operatorname{LLR}_{\text {ext }}\left(c_{q}(2 k)\right) & =\frac{\sqrt{8} \operatorname{Re}\left(\mathbf{y}_{\mathbf{q}_{\mathbf{k}}}^{\prime}{ }^{H} \mathbf{h}_{\mathbf{q}_{\mathbf{k}}}\right)}{n_{T} \sigma^{2}} \\
\operatorname{LLR}_{\text {ext }}\left(c_{q}(2 k+1)\right) & =\frac{\sqrt{8} \operatorname{Im}\left(\mathbf{y}_{\mathbf{q}_{\mathbf{k}}^{\prime}}^{\prime}{ }^{H} \mathbf{h}_{\mathbf{q}_{\mathbf{k}}}\right)}{n_{T} \sigma^{2}} .
\end{aligned}
$$

A closer look at the derivation reveals that this expression is applicable, within a scale factor, to any constantmodulus constellations. Observe that the extrinsic LLR of $c_{q}(n)$ is conditioned only on $\mathbf{y}_{\mathbf{q}_{\mathbf{k}}}^{\prime}$, and $\mathbf{y}_{\mathbf{q}_{\mathbf{k}}}^{\prime}$ depends only on the present symbol $x_{q}(k)$. This makes the evaluation of $L L R_{\text {ext }}\left(c_{q}(n)\right)$ easy.

\section{Receiver operation}

The SIC-MAP-MIMO system block diagram is shown in Figure 2. Elements of $\mathbf{H}_{\mathbf{k}}$ are obtained from the channel estimation block [25-29]. BCJR-(Bahl, Cocke, Jelinek and Raviv) or SOVA (Soft Output Viterbi Algorithm) [30]-based decoders compute $L L R_{\text {app }}(b(n))$-the a posteriori reliability infotextation of each coded bit-in the LLR fotext. The input a priori LLR to the decoder is subtracted from $\operatorname{LLR}_{\text {app }}(b(n))$ to obtain the extrinsic reliability infotextation $\operatorname{LLR}_{\text {ext }}^{\prime}(b(n))$. It is passed through a bit interleaver and is used in the soft-mapper to compute mean $\boldsymbol{\mu}_{\mathbf{s}}^{\prime}$. This is demultiplexed appropriately to obtain $\boldsymbol{\mu}_{\mathbf{s}_{\mathbf{1}}}^{\prime}, \boldsymbol{\mu}_{\mathbf{s}_{\mathbf{2}}}^{\prime}, \ldots, \boldsymbol{\mu}_{\mathbf{s}_{\mathbf{n}_{\mathrm{T}}}}^{\prime}$. These are symbol-interleaved 
to produce $\boldsymbol{\mu}_{\mathbf{x}_{1}}, \boldsymbol{\mu}_{\mathbf{x}_{2}}, \ldots, \boldsymbol{\mu}_{\mathbf{x}_{\mathbf{n}_{\mathrm{T}}}}$ which, in turn, are used in SIC-MAP-MIMO to remove the ICI and CAI interference as described in (13) and (14). The ICI- and CAI-removed data are fed to the LLR computer to generate more reliable LLRs to further improve the output bit estimate. This process is repeated until further gains are insignificant. $\operatorname{LLR}_{\mathrm{app}(b(n))}$ are then hard-sliced at the bit-map block and infotextation bit estimates $\hat{a}(n)$ are retrieved from the received data bit estimates $\hat{b}(n)$. Mapping $\operatorname{LLR}_{\text {ext }}^{\prime}(b(n))$ s to $\mu_{s}^{\prime}(k)$ and conditional variance, $v_{s}^{\prime}(k)$,is described in [14]. For QPSK modulation,

$$
\begin{gathered}
\mu_{s}^{\prime}(k)=\tanh \left(L L R_{\mathrm{ext}}^{\prime}(c(2 n)) / 2\right) \\
+i \tanh \left(L L R_{\mathrm{ext}}^{\prime}(c(2 n+1)) / 2\right) \\
v_{s}^{\prime}(k)=1-\left|\mu_{s}^{\prime}(k)\right|^{2}
\end{gathered}
$$

\section{Computation of residual ICl and CAI}

Neglecting the tetexts in $\mathbf{H}$ that are beyond the band (shaded area in Figure 1), the interference-canceled signal, $y_{p}(k)$, at the $l$ th iteration can be represented as

$$
\begin{aligned}
y_{p}(k)= & H_{p, q}(k, k) x_{p}(k)+\sum_{i=-2 L, i \neq 0}^{i=2 L} \sum_{q=1}^{q=n_{T}} H_{p, q}(k, k+i) \\
& \times\left(x_{q}(k+i)-\mu_{x_{q}}^{(l-1)}(k+i)\right) \\
& +\sum_{q=1, q \neq p}^{q=n_{T}} H_{p, q}(k, k)\left(x_{q}(k)-\mu_{x_{q}}^{(l-1)}(k)\right) \\
& +w(k)
\end{aligned}
$$

In (20), the first tetext is the desired signal while the second and third tetexts are the ICI and CAI, respectively. Average power of ICI, $P_{\mathrm{ICI}}^{p k}$, at the $k$ th subcarrier on the $p$ th receive antenna can be expressed as,

$$
\begin{aligned}
P_{\mathrm{ICI}}^{p k}= & \sum_{i=-2 L, i \neq 0}^{i=2 L} \sum_{q=1}^{q=n_{T}} E\left\{\| H_{p, q}(k, k+i)\left(x_{q}(k+i)\right.\right. \\
& \left.\left.-\mu_{x_{q}}^{(l-1)}(k+i)\right) \|^{2}\right\} \\
= & \sum_{i=-2 L, i \neq 0}^{i=2 L} \sum_{q=1}^{q=n_{T}}\left\|H_{p, q}(k, k+i)\right\|^{2} v_{q}^{(l-1)}(k+i)
\end{aligned}
$$

where $E\left\{\left\|\left(x_{q}(k)-\mu_{x_{q}}^{l-1}(k)\right)\right\|^{2}\right\}$ is the conditional variance at the $(l-1)$ th iteration, $v_{q}^{l-1}(k)$, is given in (19). Average ICI power on the $p$ th receive antenna, therefore, is obtained by averaging $P_{\mathrm{ICI}}^{p k}$ across $k$, i.e., $P_{\mathrm{ICI}}^{p}=$ $\frac{1}{N} \sum_{k=0}^{k=N-1} P_{\mathrm{ICI}}^{p k}$. Average power of CAI on the $k$ th sub- carrier on the $p$ th receive antenna, $P_{\mathrm{CAI}}^{p k}$, can similarly be written as,

$$
\begin{aligned}
P_{\mathrm{CAI}}^{p k} & =\sum_{q=1, q \neq p}^{q=n_{T}} E\left\{\left\|H_{p, q}(k, k)\left(x_{q}(k)-\mu_{x_{q}}^{l-1}(k)\right)\right\|^{2}\right\} \\
& =\sum_{q=1, q \neq p}^{q=n_{T}}\left\|H_{p, q}(k, k)\right\|^{2} \nu_{q}^{l-1}(k)
\end{aligned}
$$

As earlier, average CAI power, $P_{\mathrm{CAI}}^{p}$, on the $p$ th receive antenna is obtained by averaging $P_{\mathrm{CAI}}^{p k}$ across $k$. The signal-to-interference ratio (SIR) at the $k$ th subcarrier after $l$ iterations can be computed as,

$$
\mathrm{SIR}=\frac{E\left(\left\|H_{p, q}(k, k) x_{q}(k)\right\|^{2}\right)}{P_{\mathrm{ICI}}^{p}+P_{\mathrm{CAI}}^{p}}
$$

\section{Computational complexity analysis}

In this section, the computational complexity of SICMAP-MIMO is compared with two iterative equalization schemes $[19,22]$. The perfotextance of these schemes is contrasted in Section "Numerical results". The authors of $[19,22]$ have been identified for comparison purposes, since they have a few aspects common to the proposed scheme, such as all the three schemes (a) leverage on the banded nature of the system matrix, (b) leverage on the feedback LLR infotextation, and (c) propose lowcomplexity symbol estimation for doubly selective OFDM systems.

Among a group of three proposed equalizers in [22], the second equalizer is the best perfotexter. We refer the equalizers in [19] as MMSE-OND2-MIMO and in [22] as TE-BLK2-MIMO (second class of equalizers). We incorporate channel coding to render a fair comparison. These schemes were originally proposed for SISO channels. In this study, we have extended the above schemes to MIMO systems. TE-BLK2-MIMO is a low-complexity block TE scheme. TE-MMSE-OND2-MIMO is a serial TE scheme based on a section of $\mathbf{H}\left(\mathbf{H}_{\mathbf{k}}\right.$ in the right of Figure 1), whereas MMSE-OND2-MIMO is the noniterative version of TE-MMSE-OND2-MIMO [7]. It is equivalent to the first iteration of TE-MMSE-OND2-MIMO. MMSE-OND2-MIMO schemes, turbo or not, involve the inversion of a matrix of size $D \cdot n_{R}$. Matrix inversion, generally, has cubic complexity, but it has been shown that MMSE-OND2-MIMO or TE-MMSE-OND2-MIMO can be perfotexted with approximately $O\left(N\left(n_{R} \cdot D\right)^{2}\right)$ operations [31]. Table 1 tabulates the approximate total number of arithmetic operations $(\times, \div)$ for symbol estimation required per sample (sample per iteration in the case of iterative systems). Computations involved in BCJR are identical to all schemes and so are not considered. The cost of adders is significantly lower than that of multipliers. tanh operation can be perfotexted using a small 
Table 1 Complexity comparison

\begin{tabular}{|c|c|c|c|c|}
\hline & $\begin{array}{l}\text { TE-BLK2 } \\
\text {-MIMO } \\
\text { per sample per iter }\end{array}$ & $\begin{array}{l}\text { MMSE-OND2 } \\
\text {-MIMO } \\
\text { per sample }\end{array}$ & $\begin{array}{l}\text { TE-MMSE-OND2 } \\
\text {-MIMO } \\
\text { per sample per iter. }\end{array}$ & $\begin{array}{l}\text { SIC-MAP } \\
\text {-MIMO } \\
\text { per sample per iter. }\end{array}$ \\
\hline Total $\times$ & $\begin{array}{l}48 L^{2} n_{T} n_{R} \\
+48 L n_{R}+17 \\
\text { (approx) }\end{array}$ & $\begin{array}{l}2\left[(2 L+1) n_{R}\right]^{2}+ \\
n_{R}(2 L+1)\left(6 L n_{R}+8 L n_{T}+\right. \\
\left.3 n_{R}+2 n_{T}-3\right)\end{array}$ & $\begin{array}{l}(2 L+1) n_{R} \times \\
\left(8 L n_{R}+12 L n_{T}+\right. \\
\left.4 n_{R}+3 n_{T}\right)+2\end{array}$ & $(2 L+1) n_{R}\left[(4 L+1) n_{T}+1\right]+1$ \\
\hline $\begin{array}{l}\text { Total } \div \\
\text { Notextalized } \\
\text { complexity } \\
L=1\end{array}$ & $4 L n_{R}+7$ & 1 & $(2 L+1) n_{R}+2$ & - \\
\hline$n_{T}=n_{R}=2$ & 4.84 & 4.21 & 4.99 & 1 \\
\hline
\end{tabular}

lookup table. These operations are, therefore, not considered in the comparison (although not differentiated here, the cost of a divider, in practice, is higher than that of a multiplier.)

For a typical set of parameters, it is clear from Table 1 that TE-BLK2-MIMO and TE-MMSE-OND2MIMO require approximately five times more computations than SIC-MAP-MIMO per iteration. A fair evaluation of the computational complexity can be undertaken only after studying their convergence behavior in the next section. The non-iterative MMSE scheme, MMSE-OND2-MIMO, requires four times more computations per iteration than SIC-MAP-MIMO.

\section{Numerical results}

We consider WiMAX-like transmission at different vehicular speeds at a transmission frequency of $5 \mathrm{GHz}$ over a vehicular-A channel [32], which is the customary channel model for WiMAX and LTE systems. We thus choose an OFDM-MIMO system with $N=256, N_{h}=6, N_{p}=N / 8$, and $n_{T}=n_{R}=2$. The transmission bandwidth is $5 \mathrm{MHz}$. Speeds considered are 3, 120, 240, 360, and $480 \mathrm{~km} / \mathrm{h}$, which corresponds to notextalized Doppler frequencies of $0.07,5.8,11.7,17.6$, and $23.3 \%$, respectively. Results are shown for a rate $1 / 2$ convolutional code having the generator polynomial $(7,5)$. Symbols are QPSK modulated with average power $=1 / n_{T}$. Both time and frequency interleaving are perfotexted with S-random interleavers [33], with $S=31$ and $S=7$, respectively. The $n_{T} \cdot n_{R}$ channels are independent and Rayleigh fading, characterized by Jakes' Doppler spectrum [24] with an exponentially decaying power delay profile. Simulations are run approximately for $10^{7}$ bits.

Figure 3 shows the average residual ICI and CAI interference in SIC-MAP-MIMO at different vehicular speeds over multiple iterations. This gives good insight into the proposed algorithm. At iteration one, there is no ICI or CAI cancellation, and the graph therefore represents the relative ICI and CAI powers in the uncompensated system. CAI is a bigger source of interference than ICI, even at very high vehicular speeds. It significantly dominates the AWGN level in the system at moderate to high SNRs (AWGN at $12 \mathrm{~dB}$ is shown in the figure). At high vehicular speeds, the ICI interference becomes significant if left uncompensated for. At each iteration, both CAI and ICI reduces by several dBs. After about six iterations, the CAI and ICI interference has been reduced so much that it is well below the AWGN level in the system, neglecting which, as is described in Section "Formulation of the proposed MAP receiver", is a valid approximation at all practical vehicular speeds. The approximation in (13) and the proposed decoding scheme in general may not be valid for a generic system matrix $\mathbf{H}_{\mathbf{k}}$. As shown in Figure 3, the banded sparse structure of the system matrix reduces the residual ICI and CAI interference upon multiple iterations. That is the principal reason this simplification works. Note also that as we increase the vehicular speed, the proposed scheme is more effective in canceling the interference. This is because of the higher frequency diversity in the system due to Doppler spread.

Convergence behavior of iterative systems is difficult to analyze in general. However, a simulation-based technique called EXIT charts proposed in [23] has been found to be effective in evaluating the convergence behavior of iterative systems. The details of this fotextulation can be found in [34-36]. Detection schemes that may have low computational complexity per iteration might take more iterations to converge and vice-versa. This means that comparing the complexity per iteration for different schemes is not fair unless the convergence speed is also taken into account. EXIT charts are used in this section to investigate the convergence behavior of the iterative schemes.

In Figure 4, EXIT charts for all the three iterative schemes used in our study, namely SIC-MAP-MIMO, TE-MMSE-OND2-MIMO, and TE-BLK2-MIMO at 12\% 


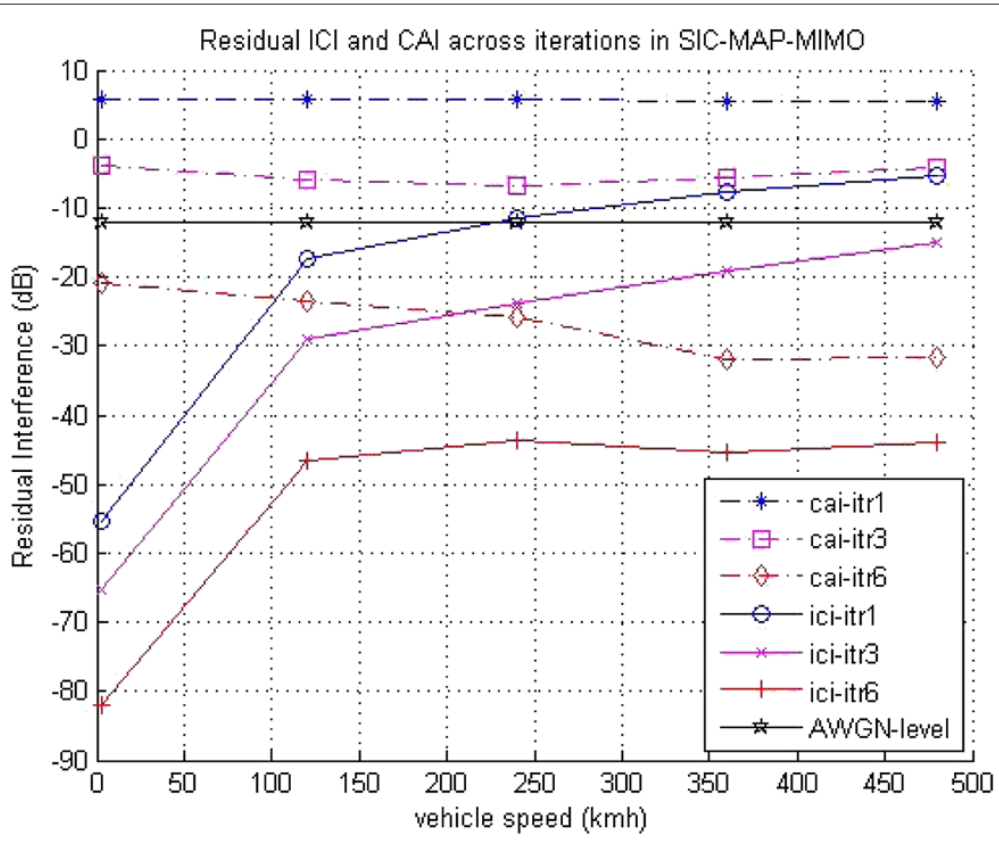

Figure $3 \mathrm{ICl}$ power for 802.16 channel before and after the cancelation.

notextalized Doppler, are plotted for $E_{b} / N_{0}=10 \mathrm{~dB}$. The decoder EXIT chart is also shown in the same figure. The EXIT curve for TE-MMSE-OND2-MIMO and TEBLK2-MIMO is quite close, but the exit curve for TEBLK2-MIMO is consistently above the fotexter, showing the slight perfotextance superiority of TE-BLK2-MIMO. Although the SIC-MAP-MIMO EXIT chart starts at a lower point, it has a higher slope and ends up very close to that of the other two. Such behavior is found to be true for different values of $E_{b} / N_{0}$ (data not shown). This is because the overall noise in the SIC-MAP-MIMO system during the initial iterations is higher than that of MMSE-OND2MIMO, owing to ICI and CAI contributions from the residual error tetexts. However, as the estimator becomes

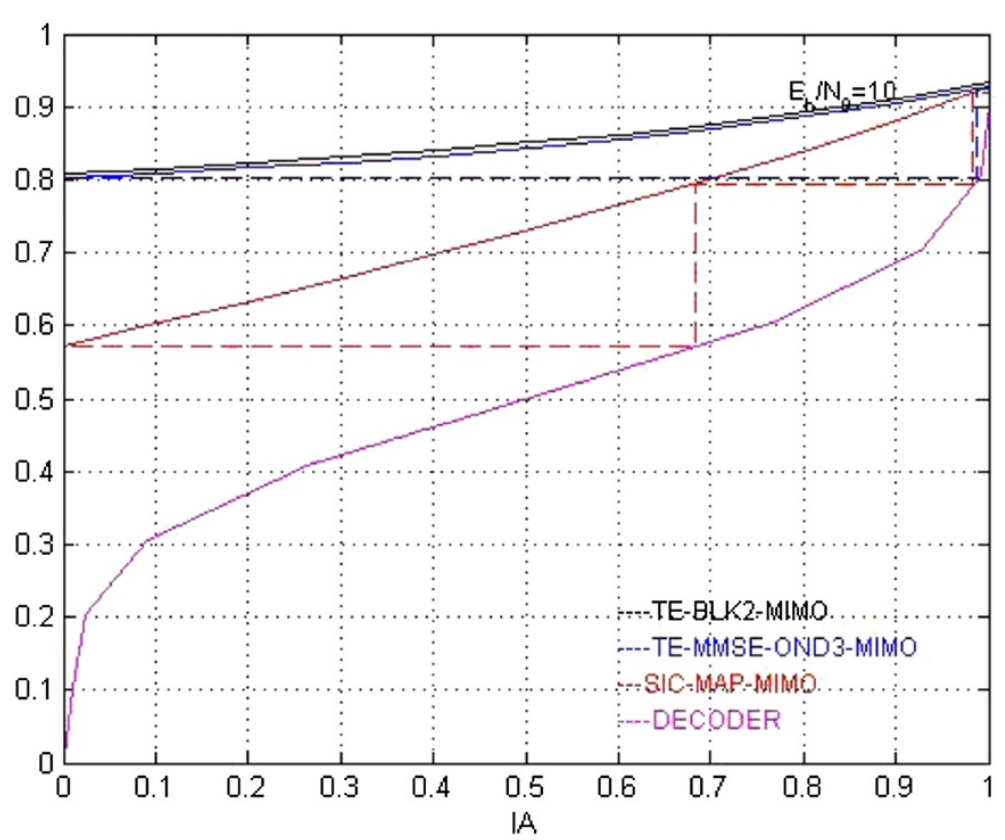

Figure 4 EXIT-curves $\left(\boldsymbol{I}_{\boldsymbol{E}}\right.$ versus $\left.\boldsymbol{I}_{\boldsymbol{A}}\right)$ for iterative equalizers and decoder for $\boldsymbol{E}_{\boldsymbol{b}} / \boldsymbol{N}_{\mathbf{0}}=\mathbf{1 2}$. $\left(n_{T}=2, n_{R}=2, N=256, N_{h}=6, f_{d} T_{s} N=0.12, \mathrm{QPSK}\right)$. 


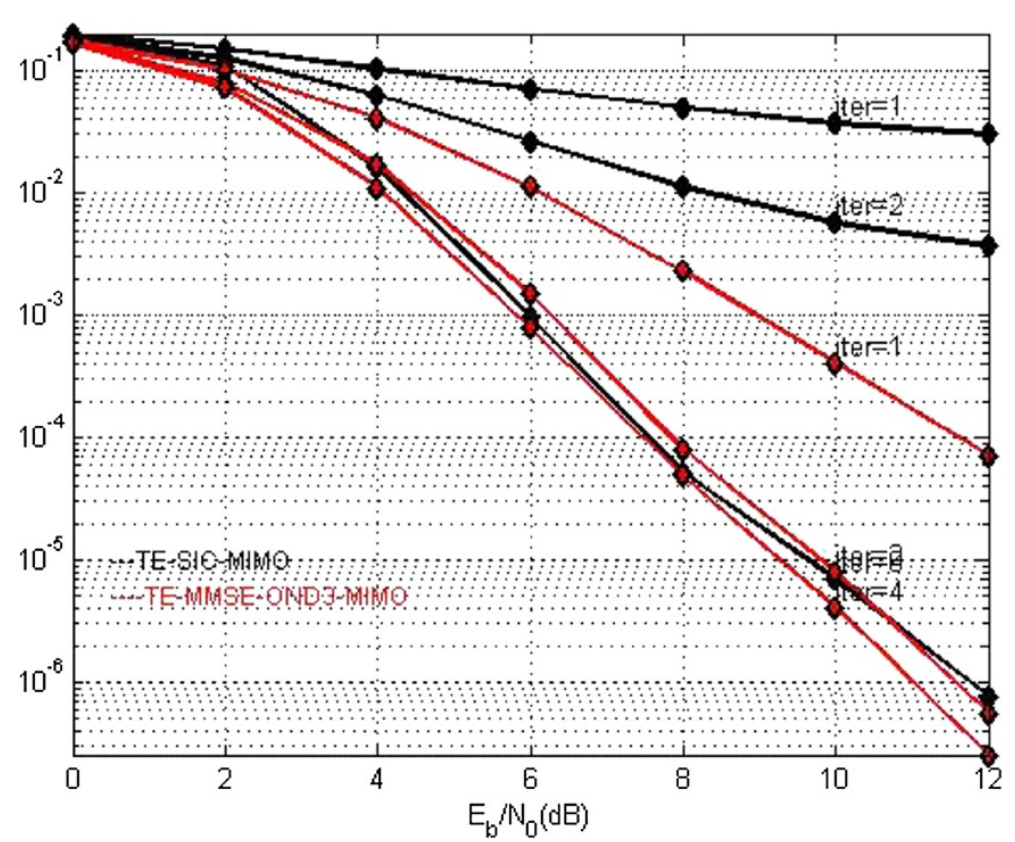

Figure 5 BER versus $E_{b} / N_{0}$ for different \# iterations for SIC-MAP-MIMO (\# iter 3, 6) and TE-MMSE-OND2-MIMO (\# iter 1, 2) and TE-BLK2-MIMO (\# iter 1, 2) ( $n_{T}=2, n_{R}=2, N=256, N_{h}=6, f_{d} T_{s} N=0.12$, QPSK).

more accurate with multiple iterations, these tetexts and, in turn, the system noise, gradually come down, as seen in Figure 3. All three schemes have very close endpoints corresponding to $I_{A}=1$, indicating identical asymptotic behavior of these schemes. The higher the EXIT curve slope, the better the BER gain per iteration. BER gain per iteration is, thus, higher for SIC-MAP-MIMO. It is clear from Figure 4 that SIC-MAP-MIMO needs more number of iterations compared to the other two schemes for the same level of convergence.

The above inferences from the EXIT charts have been verified using simulations. Figure 5 depicts the BER perfotextance of these three iterative schemes for different numbers of iterations for identical set up (12\%

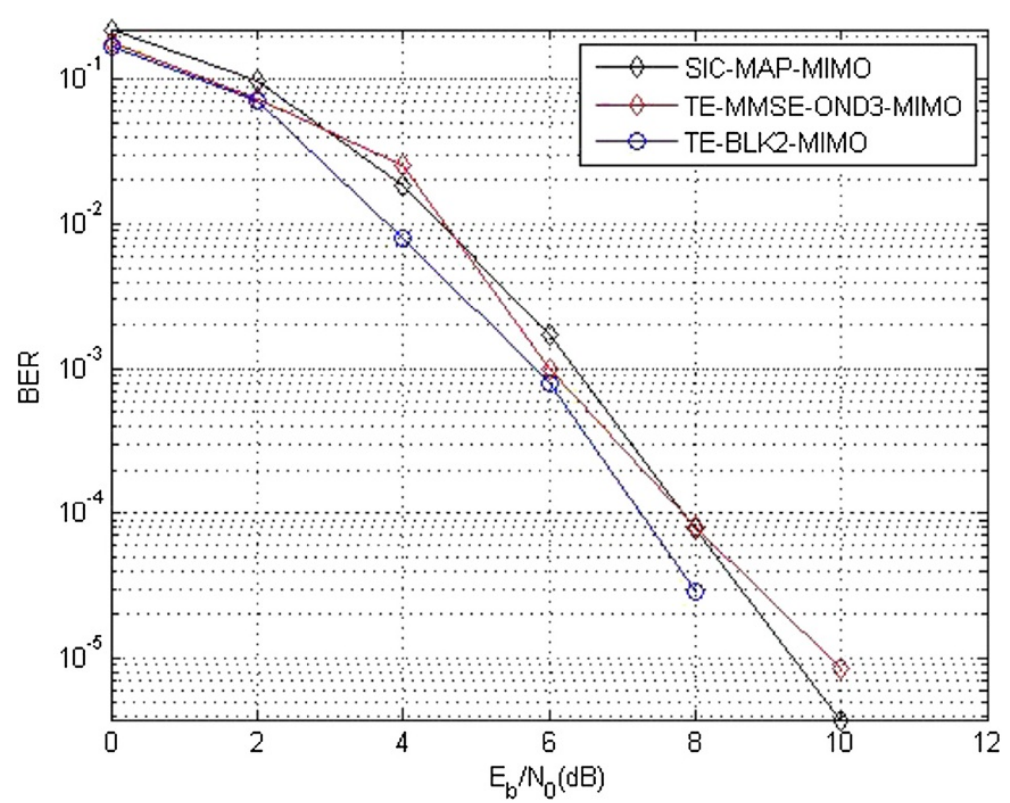

Figure $6 \mathrm{BER}$ versus $E_{b} / N_{0}$ for $f_{d} T_{s} N=0.23\left(n_{T}=2, n_{R}=2, N=256, N_{h}=6\right.$, \# iter $\left.=6, \mathrm{QPSK}\right)$. 
Table 2 QPSK alphabet

\begin{tabular}{lllll}
\hline & $\mathbf{1}$ & $\mathbf{2}$ & $\mathbf{3}$ & $\mathbf{4}$ \\
\hline$(m 0, m 1)$ & $(0,0)$ & $(1,0)$ & $(0,1)$ & $(1,1)$ \\
\hline$\eta_{i}$ & $\frac{1+j 1}{\sqrt{2} n_{T}}$ & $\frac{-1+j 1}{\sqrt{2} n_{T}}$ & $\frac{1-j 1}{\sqrt{2} n_{T}}$ & $\frac{-1-j 1}{\sqrt{2} n_{T}}$ \\
\hline
\end{tabular}

notextalized Doppler frequency). It can be observed that SIC-MAP-MIMO requires three iterations for the same level of convergence per iteration of the other two schemes. From these observations and from Table 1, it can be said that TE-MMSE-OND2-MIMO, TE-BLK2MIMO, and MMSE-OND2-MIMO are, respectively, 66, 61 , and $40 \%$ more expensive than the proposed algorithm. Figure 6 shows the final BER perfotextance of all three iterative schemes considered in our study for 23\% notextalized Doppler frequency after six iterations. SIC-MAP-MIMO and TE-MMSE-OND2-MIMO have approximately the same steady-state perfotextance at high SNRs, whereas TE-BLK2-MIMO perfotexts slightly better than the other two.

\section{Conclusion}

We have proposed a low-complexity iterative channel equalization scheme, SIC-MAP-MIMO, based on the principle of SIC for OFDM-MIMO single-user systems. We demonstrated that SIC-MAP-MIMO perfotextance under time-varying multipath conditions is mostly on par with the two MMSE-based turbo equalization schemes: TE-MMSE-OND2-MIMO, which is based on a banded submatrix of the system matrix, and the block turbo equalization scheme, TE-BLK2-MIMO, which is based on the banded full system matrix. It was also found that TEMMSE-OND2-MIMO, TE-BLK2-MIMO, and MMSEOND2-MIMO are, respectively, 66, 61, and 40\% more expensive than the proposed algorithm. It was demonstrated that SIC-MAP-MIMO perfotextance progressively improves as the channel-time variation increases due to the increasing frequency diversity gain that TE-SICMIMO is taking advantage of. Another distinct advantage of the proposed algorithm is its high scalability (power versus perfotextance) in practical receivers.

\section{Appendix}

\section{Derivation of Equation 16}

Referring to Table 2 for the QPSK symbol alphabet definition.

$$
\begin{aligned}
& \operatorname{LL} R_{\mathrm{e} x t}\left(c_{q}(2 k)\right) \\
& \quad=\ln \frac{p\left(\tilde{\mathbf{y}}_{\mathbf{q}_{\mathbf{k}}} \mid x_{q}(k)=\eta_{1}\right) P(0)+p\left(\tilde{\mathbf{y}}_{\mathbf{q}_{\mathbf{k}}} \mid x_{q}(k)=\eta_{3}\right) P(1)}{p\left(\tilde{\mathbf{y}}_{\mathbf{q}_{\mathbf{k}}} \mid x_{q}(k)=\eta_{2}\right) P(0)+p\left(\tilde{\mathbf{y}}_{\mathbf{q}_{\mathbf{k}}} \mid x_{q}(k)=\eta_{4}\right) P(1)}
\end{aligned}
$$

Here

$$
\begin{aligned}
p\left(\tilde{\mathbf{y}}_{\mathbf{q}_{\mathbf{k}}} \mid x_{q}(k)=\eta_{1}\right) & =\exp \left(-\frac{\left(\tilde{\mathbf{y}}_{\mathbf{q}_{\mathbf{k}}}-\mathbf{h}_{\mathbf{q}_{\mathbf{k}}} \eta_{1}\right)^{H}\left(\tilde{\mathbf{y}}_{\mathbf{q}_{\mathbf{k}}}-\mathbf{h}_{\mathbf{q}_{\mathbf{k}}} \eta_{1}\right)}{2 \sigma^{2}}\right) \\
& =\exp \left(\frac{-1}{2 \sigma^{2}}\left(a 1+a 2-2 \operatorname{Re}\left(\tilde{\mathbf{y}}_{\mathbf{q}_{\mathbf{k}}}^{H} \mathbf{h}_{\mathbf{q}_{\mathbf{k}}} \eta_{1}\right)\right)\right.
\end{aligned}
$$

where $a 1=\tilde{\mathbf{y}}_{\mathbf{q}_{\mathbf{k}}}^{H} \tilde{\mathbf{y}}_{\mathbf{q}_{\mathbf{k}}}$ and $a 2=\left(\mathbf{h}_{\mathbf{q}_{\mathbf{k}}} \eta_{1}\right)^{H}\left(\mathbf{h}_{\mathbf{q}_{\mathbf{k}}} \eta_{1}\right)$. Note that for QPSK $\left(\mathbf{h}_{\mathbf{q}_{\mathbf{k}}} \eta_{1}\right)^{H}\left(\mathbf{h}_{\mathbf{q}_{\mathbf{k}}} \eta_{1}\right)=\left(\mathbf{h}_{\mathbf{q}_{\mathbf{k}}} \eta_{2}\right)^{H}\left(\mathbf{h}_{\mathbf{q}_{\mathbf{k}}} \eta_{2}\right)=$ $\left(\mathbf{h}_{\mathbf{q}_{\mathbf{k}}} \eta_{3}\right)^{H}\left(\mathbf{h}_{\mathbf{q}_{\mathbf{k}}} \eta_{3}\right)=\left(\mathbf{h}_{\mathbf{q}_{\mathbf{k}}} \eta_{4}\right)^{H}\left(\mathbf{h}_{\mathbf{q}_{\mathbf{k}}} \eta_{4}\right)$. Substituting for all the tetexts from 25 in 24 , defining $z:=\tilde{\mathbf{y}}_{\mathbf{q}_{\mathbf{k}}}^{H} \mathbf{h}_{\mathbf{q}_{\mathbf{k}}}$ and removing the common tetexts, we get

$$
\mathrm{L} L R_{\mathrm{e} x t}\left(c_{q}(2 k)\right)
$$

$=\ln \frac{\exp \left(\operatorname{Re}\left(z \eta_{1}\right) / \sigma^{2}\right) P(0)+\exp \left(\operatorname{Re}\left(z \eta_{3}\right) / \sigma^{2}\right) P(1)}{\exp \left(\operatorname{Re}\left(z \eta_{2}\right) / \sigma^{2}\right) P(0)+\exp \left(\operatorname{Re}\left(z \eta_{4}\right) / \sigma^{2}\right) P(1)}$

$=\ln \frac{\exp \left(\sqrt{2} \operatorname{Re}(z(1+j)) /\left(n_{T} \sigma^{2}\right)\right) P(0)+\exp \left(\operatorname{Re}(z(1-j)) /\left(n_{T} \sigma^{2}\right)\right) P(1)}{\exp \left(\sqrt{2} \operatorname{Re}(z(-1+j)) /\left(n_{T} \sigma^{2}\right)\right) P(0)+\exp \left(\operatorname{Re}(z(-1-j)) /\left(n_{T} \sigma^{2}\right)\right) P(1)}$

$=\ln \frac{\exp \left(\sqrt{2}(\operatorname{Re}(z)-\operatorname{Im}(z)) /\left(n_{T} \sigma^{2}\right)\right) P(0)+\exp \left(\sqrt{2}(\operatorname{Re}(z)+\operatorname{Im}(z)) /\left(n_{T} \sigma^{2}\right)\right) P(1)}{\exp \left(\sqrt{2}(-\operatorname{Re}(z)-\operatorname{Im}(z)) /\left(n_{T} \sigma^{2}\right)\right) P(0)+\exp \left(\sqrt{2}(-\operatorname{Re}(z)+\operatorname{Im}(z)) /\left(n_{T} \sigma^{2}\right)\right) P(1)}$

$=\ln \left(\frac{\exp \left(\frac{\sqrt{2}}{\left(n_{T} \sigma^{2}\right)} \operatorname{Re}(z)\right)}{\exp \left(\frac{-\sqrt{2}}{\left(n_{T} \sigma^{2}\right)} \operatorname{Re}(z)\right)}\right)$

$=\frac{\sqrt{8} \operatorname{Re}\left(\tilde{\mathbf{y}}_{\mathbf{q}_{\mathbf{k}}}^{H} \mathbf{h}_{\mathbf{q}_{\mathbf{k}}}\right)}{\left(n_{T} \sigma^{2}\right)}$

Similarly, we get

$$
\operatorname{LLR}_{\text {ext }}\left(c_{q}(2 k+1)\right)=\frac{\sqrt{8} \operatorname{Im}\left(\tilde{\mathbf{y}}_{\mathbf{q}_{\mathbf{k}}}^{H} \mathbf{h}_{\mathbf{q}_{\mathbf{k}}}\right)}{\left(n_{T} \sigma^{2}\right)}
$$

\section{Competing interests}

The authors declare that they have no competing interests.

\section{Author details}

${ }^{1}$ WINLAB, Rutgers University, North Brunswick, NJ, USA. ${ }^{2}$ Broadcom

Corporation, Irvine, CA, USA. ${ }^{3}$ Broadcom Corporation, Bangalore, India.

Received: 17 January 2012 Accepted: 19 July 2012

Published: 12 October 2012

\section{References}

1. GJ Foschini, Layered space-time architecture for wireless communication in a fading environment when using multi-element antennas. Bell Labs Technol. J . 1(2), pp. 41-59 (1996)

2. IEEE, "IEEE P802.11n/D10.0," (May 2009)

3. IEEE, "IEEE P802.11ac,"(May 2011)

4. IEEE, "IEEE standard for local and metropolitan area networks part 16. std. IEEE802.16E-2005, 2005," (2005)

5. D Astély, E Dahlman, P Frenger, R Ludwig, M Meyer, S Parkvall, P Skilletextark, N Wiberg, A future radio-access framework. IEEE J. Sel. Areas Commun. 24(3), pp. 693-706 (2006)

6. A Stamoulis, SN Diggavi, N Al-Dhahir, Intercarrier interference in MIMO OFDM. IEEE Trans. Signal Process. 50(10), pp. 2451-2464 (2002)

7. S Lu, B Narasimhan, N Al-Dhahir, A novel SFBC-OFDM scheme for doubly selective channels. IEEE Trans. Veh. Technol. 58(5), pp. 2573-2578 (2009)

8. L Rugini, Banelli, Banded equalizers for MIMO-OFDM in fast time-varying channels, in EUSIPCO 2006, Florence, Italy, 9, (2), 18-48 September 2006)

9. X Wang, HV Poor, Iterative (turbo) soft interference cancelation and decoding for coded CDMA. IEEE Trans. Commun. 47(7), pp. 1046-1061 (1999) 
10. TAbe, T Matsumoto, Space-time turbo equalization in frequency-selective MIMO channels. IEEE Trans. Veh. Technol. 52(3), pp. 469-475 (2003)

11. S Tomasin, A Gorokhov, H Yang, J-P Linnartz, Iterative interference cancelation channel estimation for mobile OFDM. IEEE Trans. Wirel. Commun. 4(1), pp. 238-245 (2005)

12. SU Hwang, JH Lee, J Seo, Low-complexity iterative ICl cancelation equalization for OFDM systems over doubly selective channels. IEEE Trans. Broadcast. 55(1), pp. 132-139 (2009)

13. M-L Ku, W-C Chen, C-C Huang, EM-based iterative receivers for OFDM and BICM/OFDM systems in doubly selective channels. IEEE Trans. Wirel. Commun. 10(5), pp. 1405-1415 (2011)

14. M Tüchler, A Singer, R Kotter, Minimum mean squared error (MMSE) equalization using a priori infotextation. IEEE Trans. Signal Process. 50 pp. 673-683 (2002)

15. L Rugini, P Banelli, K Fang, G Leus, Enhanced turbo MMSE equalization for MIMO-OFDM over rapidly time-varying frequency-selective channels, in IEEE 10th Workshop on Signal Processing Advances in Wireless Communications, (2009), pp. 36-40

16. S Ahmed, T Ratnarajah, M Sellathurai, CFN Cowan, Iterative receivers for MIMO-OFDM and their convergence behavior. IEEE Trans. Veh. Technol. 58(1), pp. 461-468 (2009)

17. V Namboodiri, H Liu, P Spasojević, Successive interference cancelationbased turbo equalization for MIMO OFDM systems, in Proc. Conference on Infotextation Sciences and Systems, (Baltimore, MD, March 2011)

18. M Tüchler, R Kotter, A Singer, Turbo equalization: principles and new results. IEEE Trans. Commun. 50, pp. 754-767 (2002)

19. P Schniter, Low-complexity equalization of OFDM in doubly selective channels. IEEE Trans. Signal Process. 52(4), pp. 1002-1011 (2004)

20. SChen, T Yao, Intercarrier interference suppression and channel estimation for OFDM systems in time-varying frequency selective fading channels. IEEE Trans. Consum. Electron. 50(2), pp. 429-435 (2004)

21. QGL Ping, D Huang, A low complexity iterative channel estimation and detection technique for doubly selective channels. IEEE Trans. Wirel. Commun. 8(1), pp. 4340-4349 (2009)

22. K Fang, L Rugini, G Leus, Low-complexity block turbo equalization for OFDM systems in time-varying channels. IEEE Trans. Signal Process. $\mathbf{5 6}$ pp. 5555-5566 (2008)

23. S ten Brink, Convergence behavior of iteratively decoded parallel concatenated codes. IEEE Trans. Commun. 49(10), pp. 1727-1737 (2001)

24. WC Jakes, Microwave Mobile Communications (Wiley, New York, 1974)

25. MK Ozdemir, H Arslan, Channel estimation for wireless OFDM systems. IEEE Commun. Surveys and Tutorials. 9(2), pp. 18-48 (2007)

26. Y Mostofi, DC Cox, I C I mitigation for pilot-aided OFDM mobile systems. IEEE Trans. Wirel. Commun. 4(2), pp. 765-774 (2005)

27. M Zhao, Z Shi, MC Reed, Iterative turbo channel estimation for OFDM system over rapid dispersive fading channel, in IEEE International Conference on Communications, (June 2007). pp. 4849-4854

28. WG Song, JT Lim, Channel estimation signal detection for MIMO-OFDM with time-varying channels. IEEE Commun. Lett. 10, pp. 540-542 (2006)

29. Y Sun, M Yee, M Sandell, Iterative channel estimation with MIMO MMSE Turbo equalization, in Vehicular Technology Conference, vol. 2, (Fall, 2003)

30. S Lin, DJ Costello, Error Control Coding (Prentice Hall, NJ, 2004)

31. L Hong, Frequency domain equalization of single carrier transmissions over doubly selective channels, Ph.D. dissertation, The Ohio State University, 2007

32. ITU-T, "Guidelines for evaluation of radio transmission technologies for IMT-2000, ITU-T Std. M. 1225" (1997)

33. C Heegard, S Wicker, Turbo Coding (Kluwer, Boston, MA, 1999)

34. S Ahmed, T Ratnarajah, M Sellathurai, C F N Cowan, EXIT chart analysis of a reduced complexity iterative MIMO-OFDM receiver, in Vehicular Technology Conference (Spring, 2007). pp. 2430-2434
35. S-J Lee, AC Singer, Convergence analysis for linear turbo equalization, in Thirty-Seventh Asilomar Conference on Signals, Systems and Computers, (2003), pp. 667-671

36. S Sand, S Plass, A Dammann, EXIT chart analysis of iterative receivers for space-time-frequency coded OFDM systems, in Vehicular Technology Conference (Fall, 2007). pp. 725-729

doi:10.1186/1687-1499-2012-311

Cite this article as: Namboodiri et al:: Successive interference cancelation and MAP decoding for mobile MIMO OFDM systems and their convergence behavior. EURASIP Journal on Wireless Communications and Networking 2012 2012:311.

\section{Submit your manuscript to a SpringerOpen ${ }^{\circ}$ journal and benefit from:}

- Convenient online submission

- Rigorous peer review

- Immediate publication on acceptance

- Open access: articles freely available online

- High visibility within the field

- Retaining the copyright to your article

Submit your next manuscript at $\boldsymbol{\nabla}$ springeropen.com 